Supporting Information

\title{
Surface-charge-assisted microdroplet generation on a superhydrophobic surface
}

Fanfei Yu, ${ }^{\mathrm{a}, \mathrm{b}}$ Qiangqiang Sun, ${ }^{\mathrm{c}}$ Dehui Wang, ${ }^{\mathrm{b}}$ Yao Tan, ${ }^{\mathrm{b}}$ Shiji Lin, ${ }^{\mathrm{d}}$ Longquan Chen, ${ }^{\mathrm{d}}$ Yue Fan, ${ }^{\mathrm{b}}$ Junchang Guo, ${ }^{\mathrm{b}}$ Jinlong Yang, ${ }^{*}$ and Xu Deng a,b*

a Yangtze Delta Region Institute of University of Electronic Science and Technology of China, Huzhou 313000, P. R. China;

b Center for Materials Surface Science, Institute of Fundamental and Frontier Sciences, University of Electronic Science and Technology of China, Chengdu 610054, P. R. China;

c Key Laboratory of Advanced Technologies of Materials, Ministry of Education, School of Materials Science and Engineering, Southwest Jiaotong University, 610031 Chengdu, P. R. China; d School of Physics, University of Electronic Science and Technology of China, Chengdu 610054, P. R. China.

\section{Corresponding Author}

E-mail: jinlongy@uestc.edu.cn, dengxu@uestc.edu.cn 


\section{Contents}

Supplementary Video 1 Drop impact on SHPs with microdroplet generation

Supplementary Video 2 Drop impact on CSHPs with complete rebounding

Supplementary Video 3 Drop impact on SHPs at high velocity with undesired microdroplet generation

Supplementary Video 4 Drop impact on inclined SHPs

Supplementary Video 5 Mobility of the microdroplet trapped on SHPs

Supplementary Video 6 Grabbing the microdroplet formed on SHPs

Supplementary Figures S1-6 


\section{Supplementary Figures}
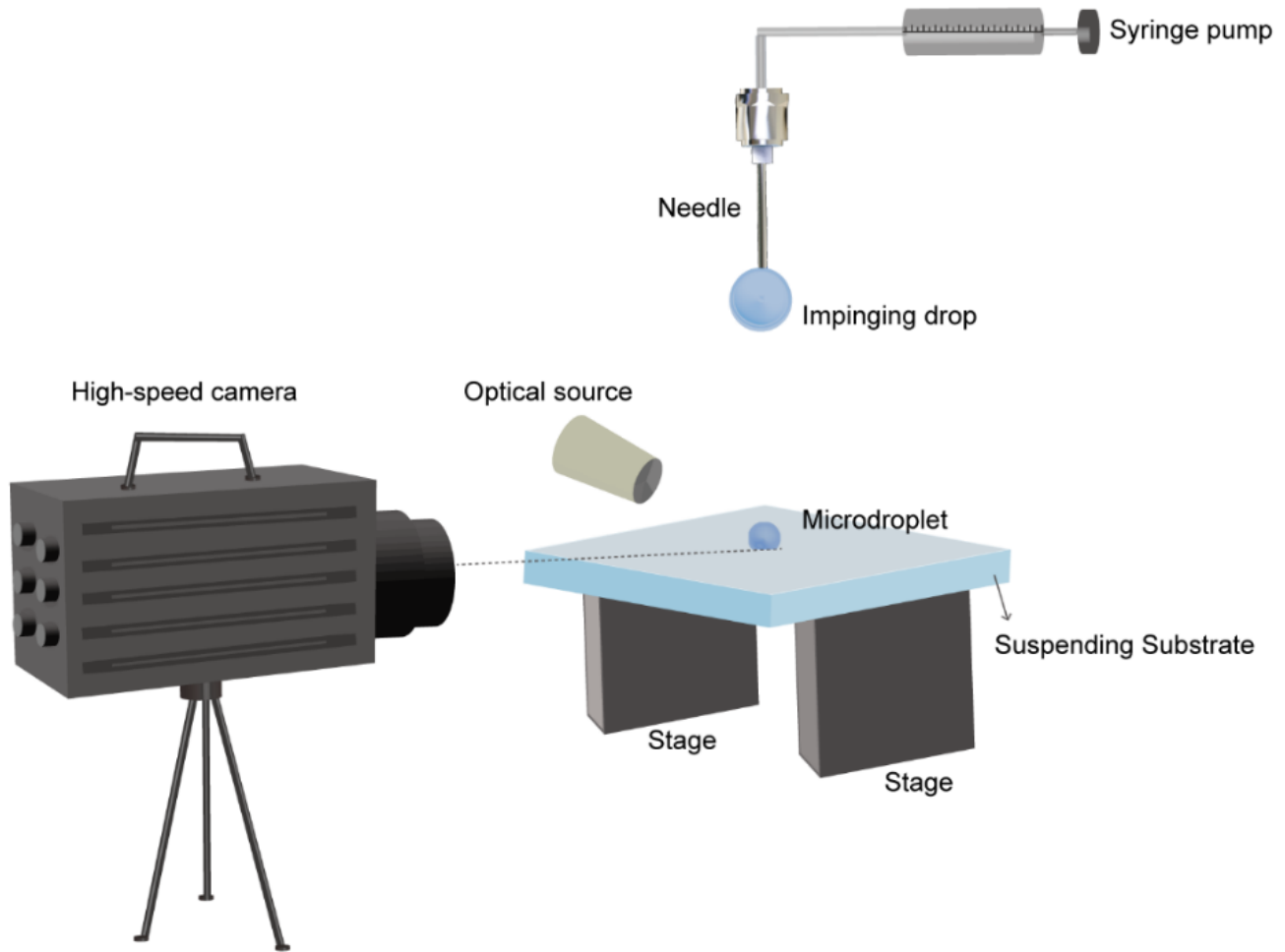

Optical source
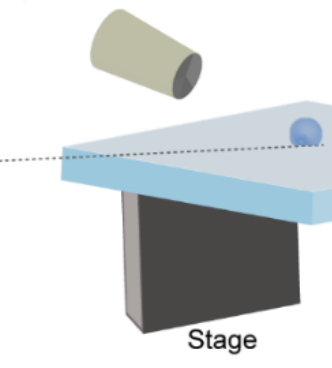

Microdroplet

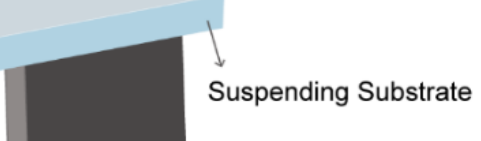

Figure S1. Experimental set-up for drop impacting. 


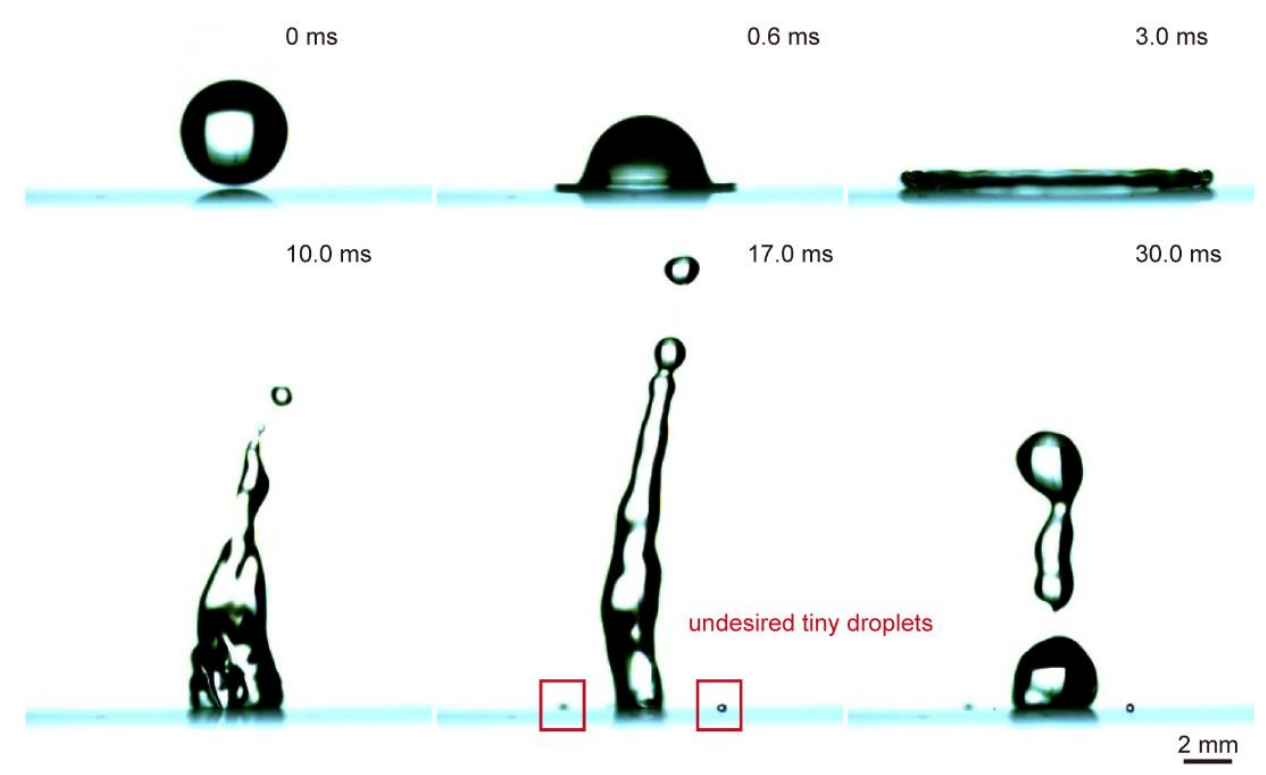

Figure S2. Undesired tiny droplets formation when drop impacting at a higher velocity (i.e., exceeding $\sim 1.7 \mathrm{~m} / \mathrm{s}$, We $\sim 99$ ) 

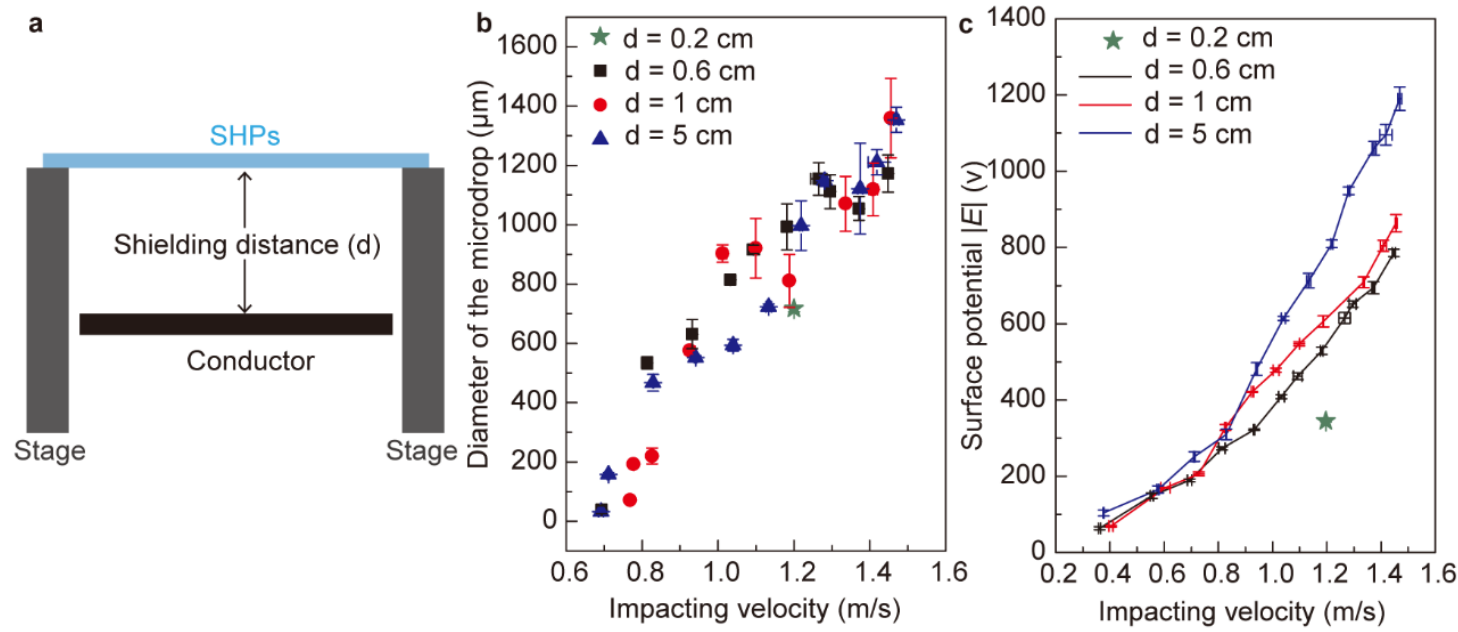

Figure S3. Influence of shielding distance on the drop impact. (a) Schematic showing the definition of the shielding distance. (b-c) Influence of shielding distance on diameter of trapped microdroplet diameter and surface potential $(E)$, respectively. 


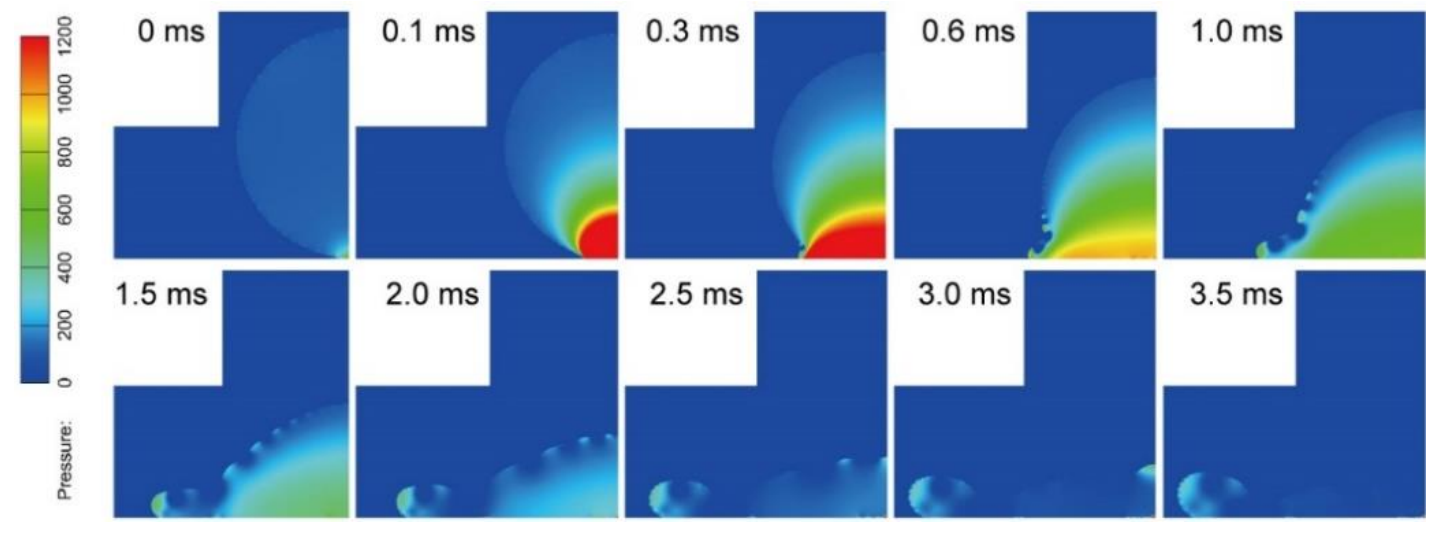

Figure S4. The pressure distribution by simulation of a drop impact on SHPs. 


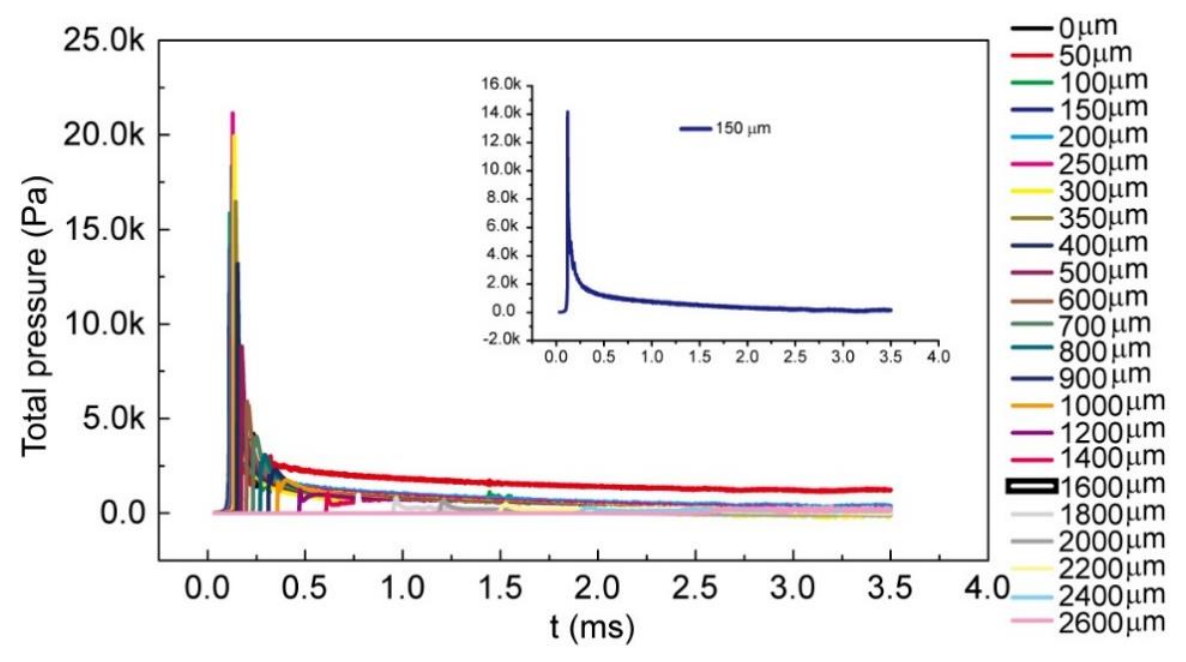

Figure S5. The total pressure monitoring during the drop impact at different position by simulation. 


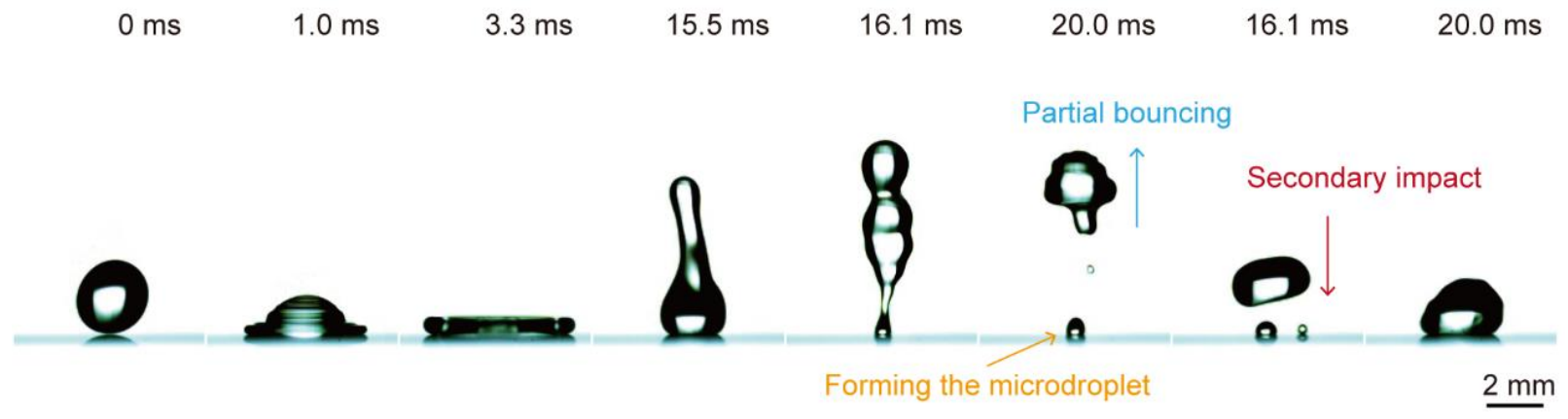

Figure S6. Secondary impact after forming the microdroplet on SHPs $(\sim 1 \mathrm{~m} / \mathrm{s})$. 